\title{
Logical Analysis of Complex Network Searching
}

\author{
Ablimit Arxiden ${ }^{1,2}$, Shanxia Wang ${ }^{1}$ and Huiwen Deng ${ }^{1, *}$ \\ ${ }^{I}$ Faculty of Computer and Information Science, Southwest University, Chongqing 400715, P.R. China; ${ }^{2}$ Faculty of \\ Mathematics and Information, Teachers Collage of Hotan, XinJiang 848000, P.R. China
}

\begin{abstract}
This article describes the relationship between network search space, search strategies and search logic with logical language. The logical property of complex network search algorithm is determined by the initial state (the search state) and the search strategy of complex network space, which can be defined logically. The article also presents the definition of complex network search states set and its relationship with first-order modal predicate logic. All possible network search state sets in the search strategy can be described with first-order modal predicate logic. Analysis of network search state properties lays a good foundation for the intelligentization of complex network search processes in the future.
\end{abstract}

Keywords: Complex network search, logical analysis, logical properties, network search state.

\section{INTRODUCTION}

Complex network search algorithm $[1,2]$ is a methodology widely used in search resources in networks. It usually proceeds as follows: firstly, the network is selected in the initial state and some network search strategies and algorithms are applied, which leads to the desired search results after a period of search. After network initial state is selected in the search process, a new state is generated under the search strategy. In the new state, it is continuously judged whether the problem is satisfied or not. This process is actually the habitual model for solving practical problems in computer science. This article, based on the algorithm modal property and relevant literature (logical analysis of search algorithm), proposes the logical relationship between the initial space of network search and the problem inquired. It also analyzes the relationship between network search process, logic reasoning, and logic satisfiable relation $[3,4]$, and points out three basic concepts, namely, derivable relationship in first-order logic, model, and satisfiable relationship covered in the network search process. It is on this basis that the logical relation between search target and network initial state space is determined. According to the logical property of search target in applying the search strategy in a complex network, a highly effective network search algorithm can be designed. The main objective of this article is to analyze the logical property of complex network search processes. The first-order modal predicate logic is used to describe the process of inquiring the target resources in complex network search algorithms. Considering relevant theories, philosophy and logical form, the logical property description and describability complex network [5] search processes are carried out. Describability complex network search processes demonstrate that the logical property of search algorithms is determined by the initial search state and the search strategy. The connection between various kinds of logic theories

*Address correspondence to this author at the Faculty of Computer and Information Science, Southwest University, Chongqing 400715, China; Te1/Fax: 023-68252352/ 023-68252051; E-mail: abtht@163.com mainly focused on the onset of models, because the grammatical derivation relation has efficacy and integrity which can be defined logically. The article once again presents a relation between the description defining complex network search state set and the first-order modal logic state set. For any possible network search state set in the search strategy, it can be characterized by the first-order predicate logic. The article consists of the following five parts: part one is the preface which briefly describes the objective; part two mainly interprets concepts related to complex network search processes and the modal predicate logic language; part three analyzes the logical property of complex network search processes; part four explains the logical property of the relation between complex network search states and search algorithms; and part five gives the conclusion.

\section{COMPLEX NETWORK SEARCH PROCESS}

In order to understand the logical property of complex network processes, a simple network search process is briefly analyzed. On a given network, there are only resources for the nodes and relations in that network, which can be searched whenever the network connection is secured. In order to better illustrate the idea, following picture is given, which is a simple social network, a teacher-and-student relation network, called TS network hereinafter. In fact, it is a very simple network consisting of three teachers, each of whom has three students, so there are nine nodes altogether. Fig. (1) shows the TS network connection and teacherstudent relations. It is a social network.

(1) Search target in network

The search target here could be a book, a phone number, an e-book, a software, a pen, a pencil, or a person (node), etc. It could also be a software or a hardware. Collectively, they comprise the target resources all of which have one thing in common: to be related to nodes, which also means that the properties of nodes are related to their properties.

(2) Problem description in network search 


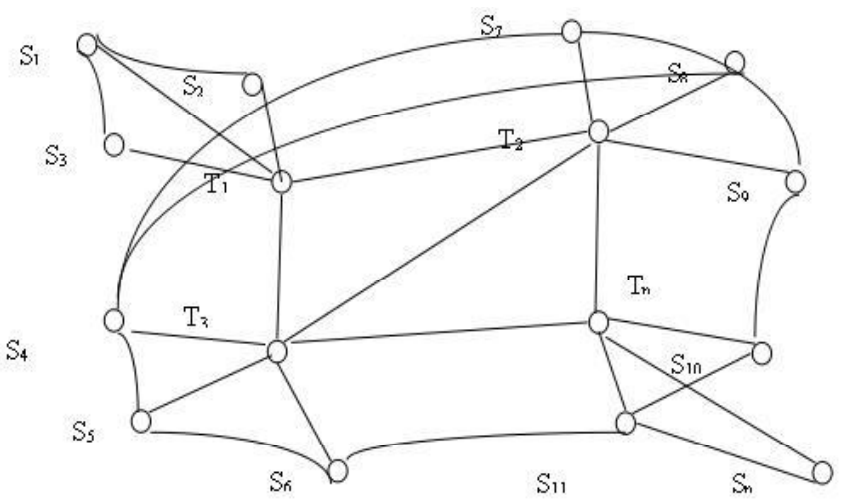

Fig. (1). TS network.

A search problem P: search of some student's phone number can also be regarded as a search problem. The same is true for searching an e-book, a textbook, or something else, etc. There are a lot of things which can be searched in the network. The process of searching any of these things is called a search problem. Suppose there are search problems $p_{1}, p_{2}, \ldots, p_{n}$, so all these search problems can be expressed as a search problem set, shown as below: $P=\left\{p_{1}, p_{2}, \ldots, p_{n}\right\}$.

(3) Network search state

Search state: it is a way of searching target resources in a network which might consists of several network search states, for example, the TS network consists of the nodes: $V=\left\{T_{1}, T_{2}, \ldots, T_{n}, S_{1}, S_{2}, \ldots, S_{n}\right\}$. The TS network's space initial state is constituted by a few search state sets: $W=\left\{W_{1}, W_{2}, \ldots, W_{n}\right\}$. The search efficiency is determined by two important factors: initial state selection and search strategy search:

$w_{1}=\left\{S_{5}, S_{4}, S_{8}\right\} ; w_{2}=\left\{S_{5}, T_{3}, T_{2}, S_{8}\right\} ;$, etc.

(4) Network search strategy

Network search strategy is a methodology in which high performance algorithms are used to enumerate all components of the solution space for a problem or all possibilities, so as to find a solution to that problem. It is the best methodology to select all or partial possibilities of searching a problem in all resources available.

Objective: there are two kinds of missions regarding search algorithms. The first is to present all possible network search states in the network search algorithm, the second is to select the best search states among all possible network search states which is the shortest search path. The one mostly concerned is how to construct target state by initial conditions and expansion rules for the process of finding the best target state (target node).

\section{(5) Network search process}

The realization of the network search process consists of two parts: the first is to control the ways of expanding nodes, the second is to expand nodes. Actually, the optimization and improvement of all search processes are completed by revising the control structure. The search strategy constantly eliminates states which cannot be searched, and chooses good search paths and searches out nodes which satisfy the search states of the problem. In an initial state, when a state meeting $P$ cannot be found after the search has begun, the search strategy might be revised to form a new search state in which the initial state meeting problem $P$ can be found. The theory here implies a strategy self-revision process in the search process.

\section{(6) Judgment of search results}

The search process could be further expressed as follows: for a search problem $P$, there are two situations regarding the conclusion or result of the initial state $w$ in a network search space: found or not found, which means the target state or target node addresses searched in target resources which are returned.

\section{LOGICAL DESCRIPTION OF COMPLEX NET- WORK SEARCH PROCESS}

Some important modal forms are also analyzed and explained such as frame, models, individuals, individual domains, etc, on the basis of the logical property of a few properties including search problem $P$, network search state, network node, network search strategy, etc. Actually, the modal predicate logic involves theoretical problems, philosophical problems, and more logical formalization and system modeling. Here it is tried to explain mainly the search process in complex networks with the modal forms of the modal predicate logic. The key is how to combine the frame with the individual domains to define the semantics of the modal predicate logic. The TS network analyzed earlier is only a small portion of the actual social network which is comprised by the relations between teachers and students. The network can be expressed as $G=\langle V, E\rangle$ in which $\mathrm{V}$ is the relation between teachers and students, $\mathrm{E}$ is the relation between teachers and teachers, teachers and students, and students and students, and so on. Here a search problem $\mathrm{P}$ can be presented, and then some search strategy can be used to solve the problem, i.e., to find the optimal search state.

Then the search process can be further expressed as follows: there is a search problem $P$, and there are two results regarding the initial state $W$ of a network search space: if found, i.e. $V(p, w)=1$ or not found, i.e. $V(p, w)=0$. In case of the former, the address of target state or target node of the target resource shall be returned. The specific process is as follows:

(1) At the beginning, the search problem $P$ can be searched in the search state $W_{1}$, in another words, the state $W_{1}$ satisfies the problem $P$, denoted by $V(p, w)=1$. 
Table 1. Relation between complex network search and the language $L$.

\begin{tabular}{|c|c|c|}
\hline Related Concepts & Complex Network Search & Modal Logic \\
\hline \hline Complex network space & $\begin{array}{c}\text { The relationship between the search state and the space of the whole net- } \\
\text { work }\end{array}$ & Frame $F$ \\
\hline Network search state & The initial state of the network space & Formula $\alpha$ \\
\hline search target & To request of search task & Model $M$ forces Formula $\alpha$ \\
\hline Network search strategy & $\begin{array}{c}\text { The application of search strategy to form a new state and decide whether } \\
\text { the problem satisfies the network search state, }\end{array}$ & \begin{tabular}{c} 
Model \\
\hline
\end{tabular}
\end{tabular}

(2) At the beginning, the search problem $P$ cannot be searched in state $W_{1}$, in another words, the state does not satisfy the problem $P$, denoted by $V(p, w)=0$.

(3) At the beginning, the search problem $\mathrm{P}$ cannot be searched in state $W$; in another words, the state $W$ does not satisfy the problem $P$, but it does not mean that the search will fail again if a different search strategy is used.

(4) The same problem P can be searched in several different search states, for example, problem $P$ can be found in the different search states $W_{1}$ and $W_{2}$. This means the two search states stand in accessible relation and the search state $W_{1}$ from $W_{2}$ can be obtained.

(5) Different search problems can be searched in the same search state.

In selecting the network search algorithm, certain models are chosen; sometimes randomly, and sometimes according to predetermined definitions. The search proceeds step by step in the time sequence, like a mechanical operation process. It is known that the logic reasoning process shares the same property. The process of inquiring the resource target is similar to satisfaction in modal logic: first, the network space initial state $W$ is determined, and the search strategy STR is decided so as to search out the target resource problem $P$. When executing the search algorithm, the process of judging whether the problem can be solved in the search state is the same as that of judging whether a modal formula is satisfied on a model, which is mainly because the search process can be described with modal predicate language. As we all know, many search functions are realized via language which essentially converts principles into description targets. Therefore, the search strategy judges the state model with the search problem as the standard for judgment. Table 1 shows the relationship between the search process of inquiring a request using a search strategy and the model of the modal predicate logic language $L$ in a complex network space.

\subsection{Logical Description of Network Search State}

The implementation of the network search process is carried by both the ways of controlling expansion nodes and the expanding of nodes. Here the optimization and improvement of all search processes are carried out by revising the control structure. The search strategy constantly eliminates network search states which cannot be searched, and constantly selects excellent network search states (network path) and searches out search state nodes according to the problem. According to the search algorithm in network search, all possible network search states comprise the model $M$ of the modal predicate logic. Here the network search problem requested equals the random equation $\alpha$ of the modal predicate logic. The network space includes the entire network search state and the relationship between search states, which is equal to the frame $F$ of the modal predicate logic. Here the relationship between network states is $R$. The modal logic language $L$ is used to describe the network search state which is to explain the network search modal form. If $\mathrm{W}$ is a network search state, then $W_{1}, W_{2}, \ldots, w_{n}$ can be used to express different network search states, and $\mathrm{W}$ is used to express the set of network search states: $W=\left\{W_{1}, W_{2}, \ldots, W_{n}\right\}$. Since there is a network search state, the natural idea is that the network search states have some node-to-node properties and relations. Different network search states have the same nodes and different nodes, and the same nodes in different network search states may also have the same properties and relations, or different ones. All nodes can be collected in any possible network search state ${ }^{W}$ into a node set $H(W)$ Here it can be explained that node of each network search state is equivalent to the explanation, property and relationship of individual and individual variable, that is the explanation of predicate symbol. If individual variable $x, y$ is $T_{1}$ and $S_{2}$ in TS network and predicate symbol $S$ and $F$ can represent the study and relationship between teachers and students respectively, then whether $S_{x}, F_{x y}$ satisfy $W$ or not, it depends on if $T_{1}$ performs the study and if $T_{1}$ is $S_{2}$ teacher: "Yes" means "satisfied" and "No" means "Not satisfied". Therefore, strictly speaking, $H(w)$ can be called the individual set of $W$. Predicate symbol is used to explain the attribute of network nodes. In this paper, Relationship $R$ between network search state $W$ 
and predicate symbol represents a frame $F=\langle W, R, D, H\rangle$, where $H \quad$ is $H(w)=\{d: d$ is an individual in $\mathrm{w}\}$ and $D$ is the universe of the discourse. All variables of $L$ take its value from $D$ and $V(x)=d$; Then the model described by the language $L$ can be expressed as a frame $M=\langle W, R, D, H, V\rangle$. Then $H$ can represent the mapping, $H: W \rightarrow \delta(D)$; It can be understood as there are individual nodes at present and some of these nodes are distributed to other possible network search states. The need is to pay attention to two points; First, regulations for revising the assignment: the domain of free variable and constraint variable shall be the same. Second, set limitation to the individual set of network search state; node set in all possible network search states shall be same as the value of explained node domain $D$. There is one kind of network search state between any two nodes in one possible network and two nodes can be found by each other. These two nodes can belong to one possible network search state and can exist in the same possible network search state. Therefore, the network search state can be defined as follows: the expression of any possible network search state is the path of certain property and relationship (attribute) from one point to another. It further explains in detail that the virtual network is just perfect to explain the concept of logical network. This point must be satisfied for the future study, that is to say, at least one property and relationship for any node in network shall be related with the property and relationship of another node. This illustrates that the registration principle of network node is connected through network. The individual in network search state must satisfy the registration principle. The attributes of each individual (node) can be considered in more detail if it is know how to select the most needed network state in many possible network search states, how to judge the performance of one certain network search state and whether it is the most needed one. In the paper, several attributes will be introduced like pheromone, hop count and route table into each node to study this problem. This problem will be discussed later after it is confirmed that how to introduce these attributes into nodes. Through the above discussion, the first order modal logical language $L$ will be formally proposed for the process of complex network search to formulate. In the logical theory, if $M$ is used to represent all model set of theoretical $L$, then all languages in $W$ can be satisfied in one of the models $W$ in $L$, with model $W$ as one sub-model of $L$. Since this model has been included in the model category, it will not make any sense to add this type of model and there is no single improvement to model category. Through comparing the model languages which satisfy the fit model, it can be judged whether this model belongs to set of models, i.e., expression of initial property in the logic [6]. The frame and model of network search state during the complex network search process can be defined as follows:
Definition 1: Assume $F=\langle W, R, D, H\rangle$ is any quadruple. $F$ is a frame of a first order modal predicate language, if $W$ is the set of network search states, $D$ is the set of nodes in network search states, both are not empty and $R$ is a binary relation on $W(R \in W \times W), H$ is a function from $W$ to $\delta(D)$.

Definition 2: (assignment) Let $F=\langle W, R, D, H\rangle \mathrm{a}$ frame and $X$ be a variable (any node) and $P$ be a predicate symbol (property and relationship of some node), $P_{x_{1}, x_{2}, \ldots, x_{n}}$ be an atomic formula, $\alpha, \beta$ be formulas and $W$ be a possible network search state in $W . V$ is an assignment on $F$, if $V$ satisfies the following condition: $V(x)=d, d \in D$ from any possible network search state $W$, it means that one corresponding node $d$ can be found in the set of nodes according to one property and relationship $V(X)$ described by any possible network search state $W$.

Definition 3: Let $M=\langle W, R, D, H, V\rangle$ be a five-tuple array network search state, $M$ is the model of one $L$. When and only when $\langle W, R, D, H\rangle$ is the frame of one $L, V$ is the assignment on this frame. This means any possible network search state $M$ is the special model while taking value $V$ from whole network search state set $F$.

Definition 4: (Satisfaction) Let $M=\langle W, R, D, H, V\rangle$ be a $L$ model and $\alpha$ be a formula including any variable $X$. $W$ is any element in $W . \alpha$ is the problem that needs to be searched:

(1) For any network search state $W \in W$, if $V(\alpha, W)=1$, then it will be said that the searching problem $\alpha$ is true on searching state $W$, denoted by ${ }_{\text {W }} \alpha$.

(2) If there exists a $W \in W$ such that $M{ }_{W} \alpha$, then it can be said that $M$ forces $\alpha$. It means problem $\alpha$ can be searched in the state $\mathrm{w}$.

\subsection{Satisfaction Problem in Network Search State}

The process of searching the resource target in network is equivalent to satisfying the relationship and determining the initial state of network space expressed as $W$. Completing the search of resource target problem $P$ through searching strategy, STR can judge if problem $P$ can be satisfied in state $W$. Of course, this satisfactory relationship is different from the classical logic, but after proper transformation, then $W \Vdash P$ is equal to $K(W) \Vdash K(p)$, where $K$ is the form transformation of sentence, meanwhile initial state is 
applied by expressing it with first order modal logic. Given two initial states $W_{1}, W_{2}$, it can be said that $\mathrm{W}_{1}$ drives $\mathrm{W}_{2}$, if the conclusion $P$ can be found through initial state $W_{2}$, the same result can be obtained by using initial state $W_{1}$. Denote the relationship between $W_{1}$ and $W_{2}$ by $W_{1} \Vdash t-W_{2}$. The notation here is to avoid the confusion in future discussion. $K$ means making judgment on the first order sentence used in the process of searching in each inquiry. Different languages have different paths in the process of judgment. Applying the transformation mapping $K$ is the more detailed definition. $K$ is used to express the searching path which can solve the problem $p$. Here it is needed to explain that there is no definite relationship between $K(p \wedge q)$ and $K(p) \wedge K(q)$, therefore there is no certain connection between the path used for solving $(p \wedge q)$ and that used for solving $p$ and $q$. The searching problem $(p \wedge q)$ in the complex network is the searching strategy and searching is carried out independently. In general, there is no connection between the searching policies of $p$ and $q$. In the future study, the construction process of the property of mapping $K(w)$ and $K(q)$ will be explained. Applying the searching strategy to set the initial state, the truth values of sentence can be judged just like the conclusion of certain problem can be obtained while applying the searching strategy to all searching processes in complex network space. Suppose the initial state is $S$, then semantics in searching process is expressed as $(\vDash)$. The grammar relation can be defined $F^{*}, F^{*} P$, which means for each model $W$ in

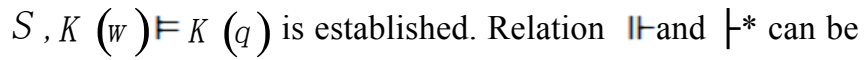
realized through $\vDash$ and $\vdash$ in classical logic. The above process is mainly used to express the logical property during complex network search through cases and logical description of complex network search process.

Model selection is made according to the initial state of network space and it further determines the complex network search algorithm. Sometimes, it is random selection and sometimes the selection can be defined in advance by person. The searching process is in steps and repeated activity according to the time sequence. The logical reasoning process is the same. The transformation process of network search is the same as the logic derivation process. Network search process analysis is used to describe the logic property of complex network search algorithm. Because initial state is the set of models and if $S$ represents the elementary class, that is sentence set $T$ is the subset of $S$. At this time, for sentence $P$, $\vdash^{*} P$ iff $T \vdash K(p)$ therefore, the conclusion from complex network search is the same as that from first order modal logic reasoning. The derivation relation $F *$ means the resource target can be obtained through network initial state when the initial searching state can express any limited sub-goal resource set.

Definition 5: (Initialization of network space state): Let $S$ be a set of initial states of network space and $T$ be the set of formulas of predicate logic language $L$, i.e., $S$ can be described by theory $T$. If $S$ can satisfy the following two conditions: (i) closed under $\Vdash$, i.e., if $w_{1} \in S, w_{1} \vdash^{*} W_{2}$, then $W_{2} \in S$. (ii) $S$ is the set of all $T^{\prime}$ models, i.e., $W_{2} \in S$ iff $T \vDash K\left(w_{2}\right)$. If $S$ satisfies the above conditions, $S$ will become an elementary class. If there is no confusion, $T \vDash K\left(W_{2}\right)$ can be written as $T \vDash W_{2}$. The closed condition means no matter how many initial states have been found in $S$, those all remain within $S$. Two initial states $W_{1}$ and $W_{2}$, as the model of predicate logic language $L$, can be derived to each other but it does not mean that they are equivalent. Suppose there is theory description for the first order modal predicate logic language in initial state set $S$, for any initial state $W$, there is always $S$ in any finite conclusion set obtained by any strategy.

\section{LOGICAL PROPERTIES ANALYSIS OF COM- PLEX NETWORK SEARCH ALGORITHM}

Searching strategy is one of the methods to exhaustedly list part of or all possible situations with definite purpose by high performance calculation method while solving the search problem. All possible situations found by searching method to find the resource target involves probably selecting the best one among all situations. Then the missions of searching algorithm are two: the first is to propose all possible network search states by the searching algorithm and the second is to select the best searching state among all possible network search states, that is, the shortest searching path. This is the problem which is to be solved. It is the process to find the best target state from the several constructed states satisfying the target nodes according to the initial state and the extension rules.

The main mission of searching algorithm is to propose all possible searching states or searching paths at first and then to select the best searching state. But the best searching state is the searching state with best searching efficiency, velocity and lowest cost from source node to target node, which is the shortest searching path. So the mission to establish the most suitable searching model definition which can satisfy the searching problem is important. Then what is the relationship between real network search state and all possible network search states? This problem can be understood like this: all possible searching states incorporate a possible situation of real searching states. In other words, there are many possible situations for real network search states. A possible situation is a possible searching state. It can be understood the other way round: there have been many possible network search states, the real network search state is no more than one state to implement the searching mission. Based on the above views, it is not necessary to put the real network search state at the prominent position, but it can be consid- 
ered as one possible network search state. Using the possible searching states to summarize all states including real network states, then it can be said that there is always one possible searching state within all possible network search states and this state is the answer to the problem. The best network search state which is the most satisfactory one for the searching process is the shortest path in the complex network search. Then how many searching states exist here actually and what kind of meaning is attributed to the possible network search states? Or what is the range of all possible network search states? The answer will be given by the working of searching algorithm: how to extend the possible network search state, what is the relationship between these possible network search states and how to connect the state with searching problem $P$ etc.

Strictly speaking, when the searching problem $P$ is considered together with a possible network search state $W$, the certainty of $p$ can be judged in $W$. Thus the "all possible network search states" is not random (all possible network search states are not considered in the actual meaning) and any possible network search state can have connection with $W$ in a certain way. Even though sometimes they are possible network search states for $W$. For $W$, it is not certain that all possible network search states are the possible network search state for $W$. Set $W$ as the possible network search state, " $S_{8}$ telephone number" which can be obtained in the realistic life. $W_{1}$ and $W_{2}$ represent the network search state for the telephone number with and without $S_{8}$ respectively, $W_{3}$ is the network search state which is never related to $S_{8}$ telephone number. For $W$, both $W_{1}$ and $W_{2}$ are the possible network search states and $W_{3}$ is the impossible network search state. According to this illustration, the certainty of problem $p$ can be considered in $W$, the impossible searching states can be ignored for $W$. Since these networking searching states are not possible for $W$ itself, no matter $p$ is true or false in these network search states, it will not influence the certainty of problem $p$ in $W$. So it can be said that one searching problem $p$ is necessarily solvable in one possible network search state, thus for $W$, it can be solved also in any possible network search state $W_{1}$. Searching can be used to judge the true value of sentence; there are two factors which impact the judgment efficiency: searching strategy and initial state. If setting of initial state is completed, the searched algorithm judges the correctness of grammar. This judgment can be operated, which means for one known sentence $\psi$, algorithm completes the judgment and obtains the detailed answer within a limited time period. The definition for this type of algorithm is equivalent to the satisfactory relationship in logic. All sentences in logic relationship have the grammar components and logic connectives which can implement the generation of sentence. Sentence composed of character string can describe the fact. In order to study the meaning of character string, it is required to define the model concept. The meaning of grammar component is interpreted before explaining the concept meaning. But this does not mean that the sentence has detailed meaning, it is to explain the meaning of sentence through judging the true or false of grammar component. Here the logical property of searching algorithm can be defined in detail as follows: the algorithm is to describe one predefined special language to describe one kind of network search process: understanding the meaning of searching, the limitation and certainty of searching are analyzed through case study, to express the process with natural simple language and then to use the predefined special language to describe the methods and procedures for solving the searching problem. Forming all network search states is equivalent to establish a frame and find out the special model of frames which satisfy the searching problem $P$.

In the study of modal logic system, many methods are established in this paper to analyze the reliability and completeness and all these methods belong to the same set. More evidences have been obtained and new derivation relationship has been obtained to replace the original theory. The modal logic property on theory in modal logic and searching algorithm property are related with the same model set. From the view of logic theory, $M$ is all model sets of $L$, then when all satisfactory languages in $W$ can be satisfied in a model $W$ of $L$, model $W$ is one subset of model category $L$. Therefore it will not make any sense to add this type of model anymore and there is no single improvement to model category. Through comparing the model languages which satisfy the fit model, it can be judged whether this model belongs to the model set. Nevertheless, in searching algorithm, the answer of problem $P$ can be obtained through network search state. Therefore, the following conditions need to be satisfied: initial network search states set shall include the required network search states. In addition, when applying the modal predicate logic language to describe, the final reasoning result is the conclusion from the searching algorithm. Detailed description for the relationship between network search state and network search states set is given in this paper. States set can be obtained by describing the modal predicate logic property. Mathematical logic is very important tool to study the computer technology and artificial intelligence. In this paper, it is tried to explain the logical application in the process forming of complex network search. Based on the above analysis, the relationship between complex network search and logic can be summarized into three types: standardization; programming; verification.

\section{CONCLUSION}

The first order modal predicate logic is used in this paper to describe the process of searching the target resource by complex network search algorithm. Taking the complex network search process as the object, the logic property description of complex network search process from the aspects of theory, philosophy and logic form is considered. The describability of network search decides the logic property of searching algorithm that depends on the initial network search sate and network search strategy. At first, the 
relationship between searching process and logic reasoning is given and the equivalence between searching states set and model set. The connection among various logic theories is mainly represented by the model set, this is because grammar derivation relationship has effectiveness and completeness, which can be defined under the sense of logic. Secondly, the description to define the complex network search state set and the relationship between first order modal and this logic state set are proposed. All possible network search states set in the searching strategy can be described by first order modal predicate logic.

\section{CONFLICT OF INTEREST}

The authors confirm that this article content has no conflict of interest.

\section{ACKNOWLEDGEMENTS}

This work was supported by the Major Project of National Social Science of China "A Logical, Cognitive and Computational Study on information interaction "(14ZDB016).

\section{REFERENCES}

[1] D.J. Watts, and S.H. Strogatz, "Collective dynamics of small-world networks," Property, vol. 393, no. 6684, pp. 440-442, 1998.

[2] W. Chen, F. Gong, Y. Li, "New strategy of resource searching in unstructured p2p network," International Conference On Communication Software \& Networks, 2010, pp. 32-36.

[3] E. A. Emerson, Temporal And Modal Logic, Handbook of Theoretical Computerence, The MIT Press, Cambridge, 1995.

[4] V. Goranko, and M. Otto, "Model theory of modal logic," Studies In Logic \& Practical Reasoning, vol. 3, no. 7, pp. 249-329, 2007.

[5] A. L. Barabási, and R. Albert, "Emergence of scaling in random networks," Science, vol. 286. no. 5439, pp. 509-512, 1999.

[6] Blackburn P, Rijke M D, Venema Y. Modal Logic" Cambridge University Press, UK, 2002.

(C) Arxiden et al.; Licensee Bentham Open.

This is an open access article licensed under the terms of the (https://creativecommons.org/licenses/by/4.0/legalcode), which permits unrestricted, noncommercial use, distribution and reproduction in any medium, provided the work is properly cited. 